Case Report

\title{
Candida glabrata Pneumonia in a Patient with Chronic Obstructive Pulmonary Disease
}

\author{
Onur Yazici, ${ }^{1}$ Mustafa Cortuk, ${ }^{2}$ Hasan Casim, ${ }^{3}$ Erdogan Cetinkaya, ${ }^{2}$ \\ Ali Mert, ${ }^{4}$ and Ali Ramazan Benli ${ }^{5}$ \\ ${ }^{1}$ Department of Chest Disease, Adnan Menderes University, Aydin, Turkey \\ ${ }^{2}$ Department of Chest Disease, Karabuk University, Karabuk, Turkey \\ ${ }^{3}$ Department of Chest Disease, Karabuk University Training and Research Hospital, Karabuk, Turkey \\ ${ }^{4}$ Department of Infectious Diseases, İstanbul Medipol University, İstanbul, Turkey \\ ${ }^{5}$ Department of Family Medicine, Karabuk University, Karabuk, Turkey
}

Correspondence should be addressed to Mustafa Cortuk; mcortuk@yahoo.com

Received 18 June 2016; Revised 21 September 2016; Accepted 16 October 2016

Academic Editor: Sandeep Dogra

Copyright (C) 2016 Onur Yazici et al. This is an open access article distributed under the Creative Commons Attribution License, which permits unrestricted use, distribution, and reproduction in any medium, provided the original work is properly cited.

Pneumonia remains an important cause of morbidity and mortality among infectious diseases. Streptococcus pneumoniae and viruses are the most common cause of pneumonia. Candidiasis in such patients has been associated with haemodialysis, fungal colonization, exposure to broad-spectrum antibiotics, intensive care unit (ICU) hospitalization, and immunocompromised patients. The most common cause of infection is C. albicans. The case presented here is of a 66-year-old male patient diagnosed with C. glabrata. The patient suffered from chronic obstructive pulmonary disease.

\section{Introduction}

Pneumonia is a common respiratory tract disease and is one of the leading causes of mortality. Streptococcal and viral pneumonia are determined to be among the most common causes of community-acquired pneumonia in adults. In individuals with normal immunity, Candida species rarely cause pneumonia. The most common pathogen among the Candida species is $C$. albicans, particularly in subjects with reduced immunity or patients necessitating intensive care management [1].

C. glabrata is known as a nonpathogen Candida species. C. glabrata rarely acts as an infectious agent when compared to other Candida species and it is present within normal respiratory flora [2]. Few cases have been reported in which C. glabrata was determined to be an infectious agent causing pneumonia [3-6].

In this case report, C. glabrata pneumonia was diagnosed in a patient who has chronic obstructive pulmonary disease (COPD) and has been taking thyroid replacement therapy.

\section{Case Presentation}

A 66-year-old male patient was admitted with cough, purulent and blood-mixed sputum, and increased shortness of breath. His medical history included COPD for the last 5 years and total thyroidectomy performed 3 years ago with the diagnosis of multinodular goiter. When questioned about prescriptions, he was under inhaled formoterol, ipratropium bromide, and levothyroxine sodium, administered orally at $100 \mu \mathrm{g} /$ day. He had not been hospitalized in the last year. Physical examination revealed blood pressure of $110 / 70 \mathrm{mmHg}$ and pulse of $89 /$ minute; his temperature was $36.8^{\circ} \mathrm{C}$ and fingertip-measured oxygen saturation $\left(\mathrm{SpO}_{2}\right)$ was $89 \%$ on room air. He presented with decreased breath sounds and prolongation of expiratory time. In addition, rales were present on auscultation of the mid and lower zones of the lungs. Laboratory investigation revealed C-reactive protein of $85 \mathrm{mg} / \mathrm{dL}$, leukocytosis, with a white blood cell (WBC) count of $12.000 / \mathrm{mm}^{3}$, and increased erythrocyte sedimentation rate $(35 \mathrm{~mm} /$ hour $)$. The chest X-ray showed increased bronchovascular shadows of the lungs. Treatment 


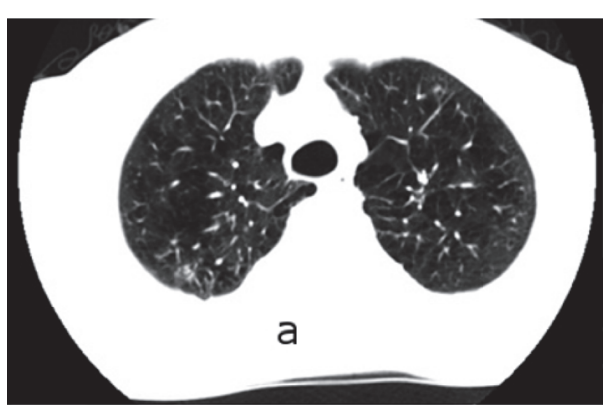

(a)

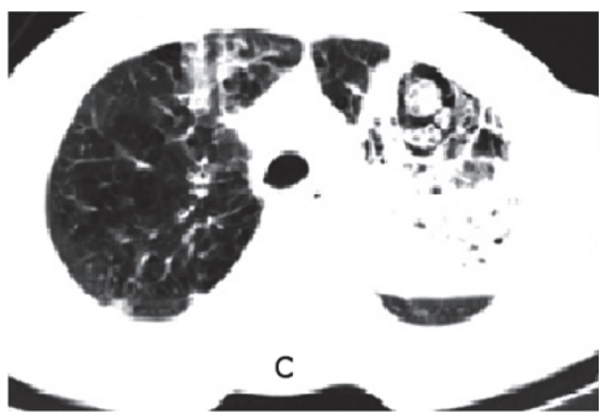

(c)

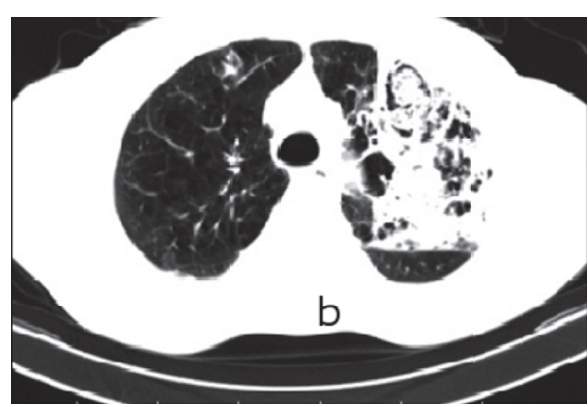

(b)

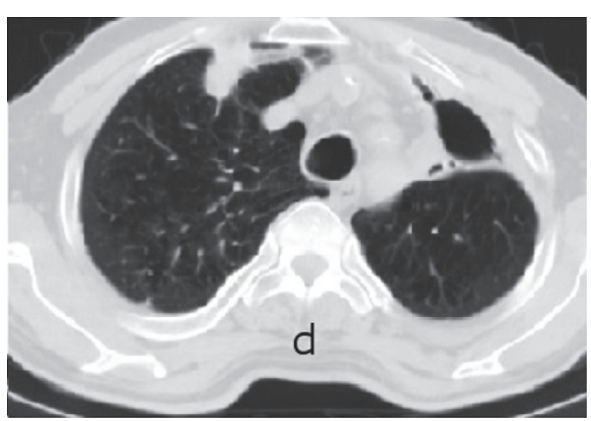

(d)

FIGURE 1: Chest tomography views on 4th day (a), 18th day (b and c), and 6th month (d) of hospitalization.

of an inhaler bronchodilator, intravenous theophylline, and $40 \mathrm{mg} /$ day methylprednisolone was started. An antibiotic combination of intravenous ampicillin sulbactam ( $4 \mathrm{~g} /$ day) and oral levofloxacin (500 mg/day) was administered. On the 4 th day of treatment, the administered antibiotics were discontinued due to a deterioration in clinical features together with progression of infection parameters and cefoperazone sodium/sulbactam sodium combination ( $4 \mathrm{~g} /$ day) and moxifloxacin ( $400 \mathrm{mg}$ /day) were started as new antibiotics. Computerized tomography (CT) of the chest was performed where emphysema and a small infiltration of right upper lobe posterior part were identified (Figure 1(a)). As the patient's clinical condition was getting worse and the infection parameters were not improving despite the treatment, a chest Xray was obtained, which showed an increase of nonhomogeneous density on the left upper lobe. Additionally, flexible bronchoscopy was performed with normal findings except increased bronchial submucosal vascularity on the tenth day of being admitted to the hospital. Streptococcus mitis and Candida species were produced in the bronchoalveolar lavage obtained by left upper lobe. Therefore, the antibiotics were discontinued and linezolid $800 \mathrm{mg} /$ day and fluconazole $200 \mathrm{mg} /$ day were started intravenously. Meanwhile, an increase in blood sugar level was detected, which was considered to be related to intravenous corticosteroid. Therefore, the corticosteroid dosage was reduced and discontinued on the 12th day, after which the blood sugar level returned to normal. Since $\mathrm{SpO}_{2}$ continued to remain below $88 \%$ despite administration of $5 \mathrm{~L} / \mathrm{min}$ oxygen and tachycardia and tachypnea were present, the patient was admitted to the intensive care unit and bilevel positive airway pressure (BPAP) treatment was applied. Despite this treatment, no improvement was observed in the patient. A further chest CT was obtained which showed a pneumonic appearance with patchy necrotic areas in the left upper lobe (Figures 1(b) and 1(c)). Flexible bronchoscopy was performed again on the 25th day of being admitted to the hospital and it revealed a view consistent with diffuse white Candida plaques in sites starting from the vocal cords and covering the entire trachea and both bronchial systems (Figure 2(a)). Bronchial biopsies and lavage of the left upper lobe were obtained. The bronchial biopsy revealed no fungus or similar pathology in the tissue. Because Candida spp. is reproduced in bronchoalveolar lavage taken at both 10th and 25th days, and Candida plaque is compatible with macroscopic appearance on bronchoscopy done at 25th day, it has been thought to be resistant Candida spp. and voriconazole treatment has been started. The bronchoalveolar lavage sample that Candida spp. reproduced on Candida plaques that are taken from BAL sample was sent to "Refik Saydam National Public Health Agency" for classification and antibiogram. Fluconazole resistant $C$. glabrata reproduced on the fungi culture that is done in this institution. The MIC90S value was reported as $\geq 32 \mu \mathrm{g} / \mathrm{mL}$ for fluconazole and $1 \mu \mathrm{g} / \mathrm{mL}$ and for voriconazole. We could not obtain Candida specific antibodies and mannan antigen tests that are used for the diagnosis of Candida infections, because it is not performed in our hospital. The fluconazole was discontinued and intravenous voriconazole $400 \mathrm{mg} /$ day was started. Following this treatment, the clinical status of the patient gradually ameliorated, hemoptysis stopped, 
and infection parameters improved. The patient, who no longer required BPAP, was admitted to the clinical ward. Intravenous voriconazole was continued for 23 days. Control bronchoscopy performed on the 15 th day of voriconazole treatment revealed almost complete recovery of the previously observed Candida plaques. Following significant improvement of his general status and dyspnea, the patient was discharged from the hospital with a prescription for oral voriconazole. Blood and urine cultures obtained during the hospitalization did not manifest any Candida growth. During ambulatory treatment with voriconazole, E. coli grew once in the urine culture and this was treated with ertapenem administered for 10 days. The voriconazole treatment was continued for 6 months. The appearance of air-fluid level at the left upper lobe and traction of the trachea to the left were identified during treatment. Left upper lobectomy was recommended because of this radiological appearance, but the patient refused surgery and so was continued to be followed up clinically and radiologically. The clinical situation of the patient did not worsen. Radiological and bronchoscopic recovery (Figure 2(b)) was seen on the last obtained CT (Figure 1(d)). Therefore, the idea of surgery was abandoned and the ambulatory follow-up of the patient currently continues uneventfully.

\section{Discussion}

Candida species exist as opportunistic pathogens in the microflora of the human body [7]. Candida infections are encountered especially in patients hospitalized in intensive care units (ICU) and these infections prolong ICU stay and increase the mortality rate [8]. When Candida is produced, particularly in cases obtained from the respiratory tract, the most important problem is due to either colonization or invasive pulmonary candidiasis. Since Candida is an element of microflora, to differentiate from colonization, it is necessary to be identified in the tissue, in general.

Pulmonary fungal infections are being increasingly identified as infectious agents in immunocompromised subjects. In the article by Chen et al., in which pulmonary fungal infections were compiled, C. glabrata was reported in 4 out of 140 patients [9].

Candida pneumonia is caused either by candidemia via the hematogenous route or by aspiration from the oropharynx. Kobayashi et al. reported the case of a 71-year-old patient who was being fed through nasogastric catheter [3]. In this case, the probable contamination route was considered to be aspiration. Another 78-year-old patient, reported by Speletas et al., had chronic myeloid leukemia and was using imatinib mesylate [6]. In this patient, candidemia was not reported. Hamilton et al. reported a case of Candida glabrata pneumonia with candidemia in an immunocompetent patient [5]. Bankier et al. reported a case of C. Glabrata infection in a nonimmunocompromised patient [4]. To the best of our knowledge, no other case has been reported except those mentioned above.

Franquet et al. reported that there is no specific radiologic image on Candida pneumonia. It has been reported that, in the study, computerized tomography (CT) findings included multiple nodules and air-space consolidation and nodules surrounded by discrete areas of ground-glass opacity (CT halo sign) can be seen [10]. In our case consolidation and cavitation were also shown on the CT of left upper lobe and a nodule of right upper lobe. Although there are many respiratory signs and symptoms from the respiratory system on the admission day, the patients suffered from COPD for the last 5 years.

In the current case, the patient suffered from COPD and thyroid replacement therapy. There was no history of central catheter utilization or admission to hospital or intensive care unit. There was no history of vomiting and aspiration. Although the lower respiratory tract infection is a common complication of COPD that is being presented in the case, general immune deficiency has not been determined. Although colonization by Candida of multiple nonsterile sites has been declared, prolonged use of antibacterial antibiotics has been linked to increased risk of invasive candidiasis [11]. In our case, Candida plaques were seen under the vocal cords which were excepted as sterile normally. Also, on the laboratory parameter, CRP sedimentation and WBC were found to be high on the day when the patient is hospitalized and these parameters got worse despite the antibiotics. The status of the patient deteriorated from the beginning and no response was observed from the administered nonspecific treatments. Since the second bronchoscopy, which was performed while the patient was under empirical fluconazole treatment, revealed Candida plaques in the entire bronchial system starting from the vocal cords, the fluconazole treatment was discontinued before the culture results were obtained and voriconazole was started. Thereafter, the culture result was received as C. glabrata and its resistance against fluconazole was verified by antibiogram. Previous publications have frequently identified the resistance of C. glabrata to azole group antifungals [12].

To differentiate a pulmonary infection caused by Candida from colonization, the microbiological agent should be identified in tissue, in general. In the current case, we showed wide Candida plaque on the bronchoscopy, but, unfortunately, we could not identify it microscopically in the tissue. Nevertheless C. glabrata were identified by bronchoalveolar lavage twice. Although Aspergillus type fungi were intensively sought in both the biopsy specimens and the cultures, due to a similar radiological appearance, they were not identified.

\section{Conclusion}

C. glabrata is quite a rare cause of pneumonia. Although it is quite rare, it may also be the cause in ambulatory patients. It should be kept in mind especially in cases where the clinical condition does not improve despite treatment with azole group antifungal agents and Candida species are detected in respiratory tract isolates. It should also be kept in mind that successful treatment is possible with voriconazole.

\section{Consent}

Written informed consent was obtained from the patient. 


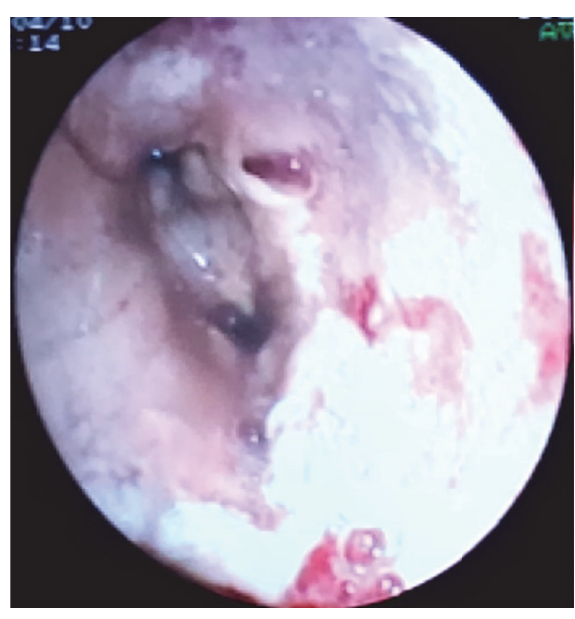

(a)

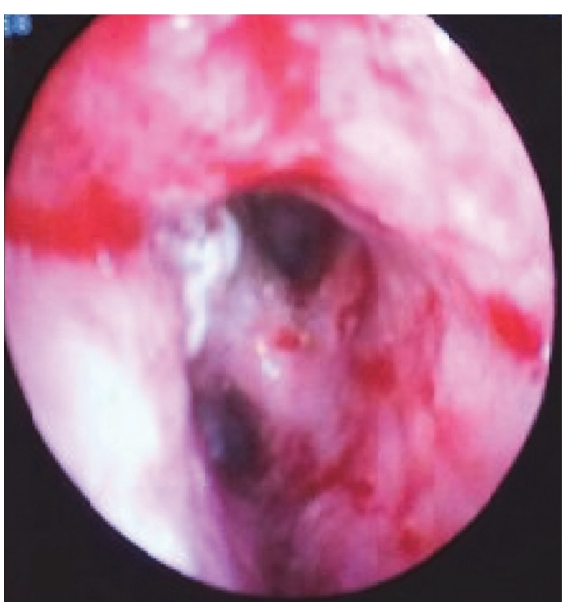

(b)

Figure 2: The appearance of the left main bronchus in the bronchoscopic examination performed before (a) and after (b) the 15th day of voriconazole treatment.

\section{Competing Interests}

No competing interests were declared by the authors.

\section{References}

[1] S. Li and Y.-Z. An, "Retrospective analysis of invasive fungal infection in surgical intensive care unit," Zhonghua Yi Xue Za Zhi, vol. 90, no. 6, pp. 382-385, 2010.

[2] L. D. Haley, "Yeasts of medical importance," American Journal of Clinical Pathology, vol. 36, pp. 227-234, 1961.

[3] T. Kobayashi, Y. Miyazaki, K. Yanagihara et al., "A probable case of aspiration pneumonia caused by Candida glabrata in a nonneutropenic patient with candidemia," Internal Medicine, vol. 44, no. 11, pp. 1191-1194, 2005.

[4] A. Bankier, D. Fleischmann, M. Wiesmayr, K. Laczika, and P. Hübsch, "Candida glabrata pneumonia in a non-immunosuppressed patient: diagnostic imaging with digital luminescence radiography and CT," Aktuelle Radiologie, vol. 4, no. 4, pp. 192194, 1994.

[5] L. A. Hamilton, N. R. Lockhart, and M. R. Crain, "Candida glabrata and Candida tropicalis in an immunocompetent patient: a case report," Journal of Pharmacy Practice, vol. 28, no. 3, pp. 284-287, 2015.

[6] M. Speletas, T.-A. Vyzantiadis, F. Kalala et al., "Pneumonia caused by Candida krusei and Candida glabrata in a patient with chronic myeloid leukemia receiving imatinib mesylate treatment," Medical Mycology, vol. 46, no. 3, pp. 259-263, 2008.

[7] E. Azoulay, J.-F. Timsit, M. Tafflet et al., "Candida colonization of the respiratory tract and subsequent pseudomonas ventilator-associated pneumonia," Chest, vol. 129, no. 1, pp. 110117, 2006.

[8] N. Adigüzel, Z. Karakurt, G. Güngör et al., "Mortality rates and risk factors associated with nosocomial Candida infection in a respiratory intensive care unit," Tuberküloz ve Toraks, vol. 58, no. 1, pp. 35-43, 2010.

[9] K.-Y. Chen, S.-C. Ko, P.-R. Hsueh, K.-T. Luh, and P.-C. Yang, "Pulmonary fungal infection: emphasis on microbiological spectra, patient outcome, and prognostic factors," Chest, vol. 120, no. 1, pp. 177-184, 2001.
[10] T. Franquet, N. L. Müller, K. S. Lee, A. Oikonomou, and J. D. Flint, "Pulmonary candidiasis after hematopoietic stem cell transplantation: thin-section CT findings," Radiology, vol. 236, no. 1, pp. 332-337, 2005.

[11] P. G. Pappas, J. H. Rex, J. D. Sobel et al., "Guidelines for treatment of candidiasis," Clinical Infectious Diseases, vol. 38, no. 2, pp. 161-189, 2004.

[12] I. I. Balkan, A. Savas, A. Geduk, M. Yemisen, B. Mete, and R. Ozaras, "Candida glabrata perinephric abscess," The Eurasian Journal of Medicine, vol. 43, no. 1, pp. 63-65, 2011. 


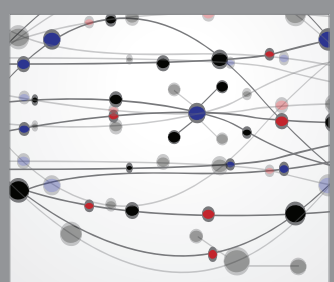

The Scientific World Journal
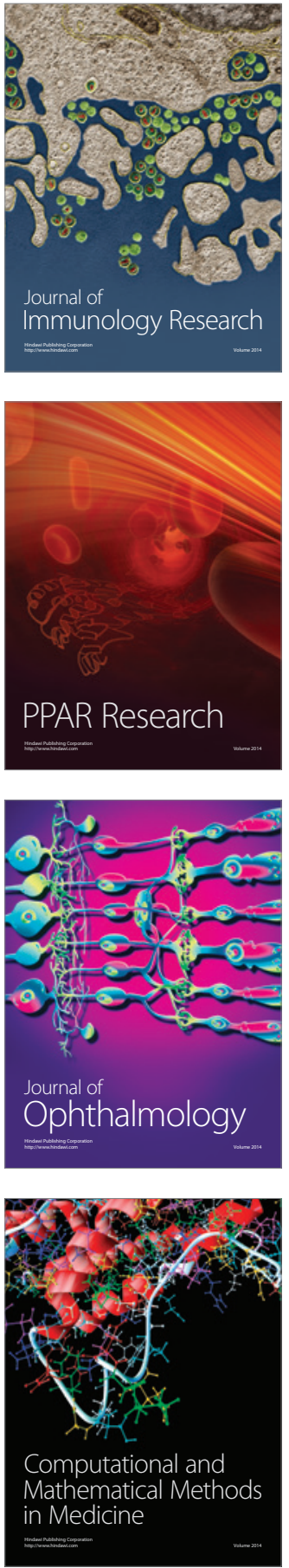

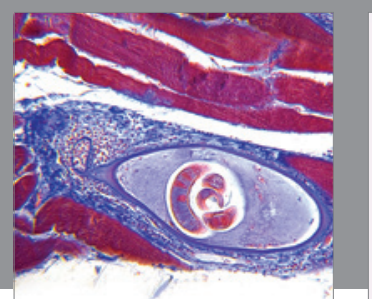

Gastroenterology Research and Practice

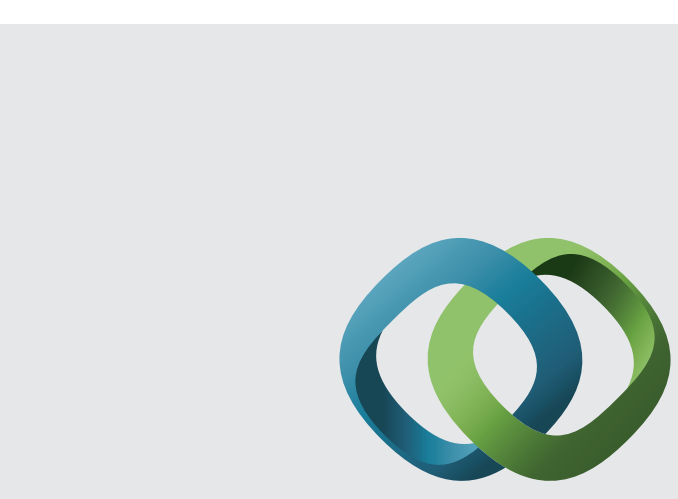

\section{Hindawi}

Submit your manuscripts at

http://www.hindawi.com
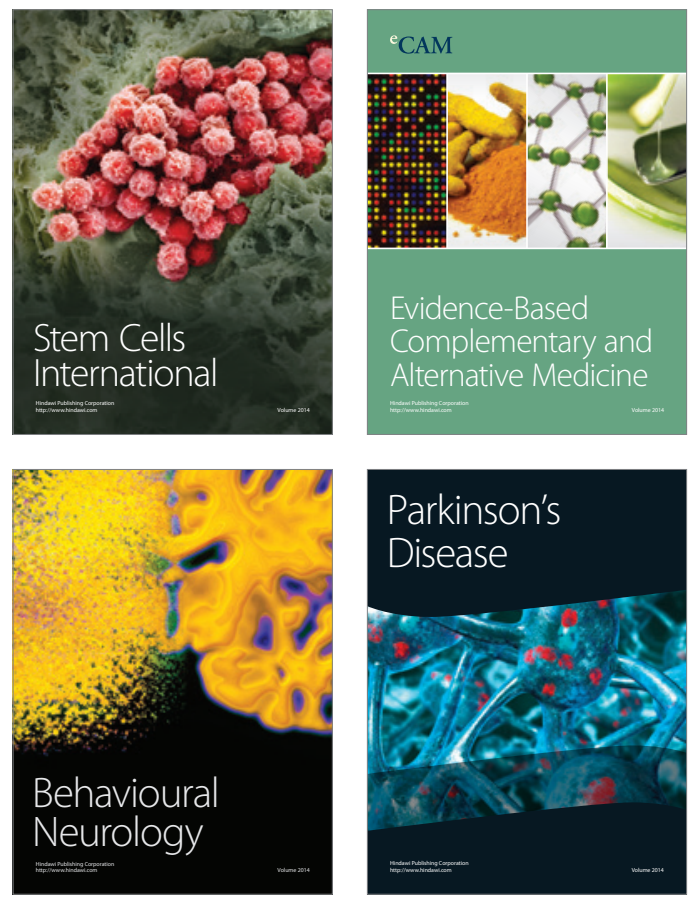
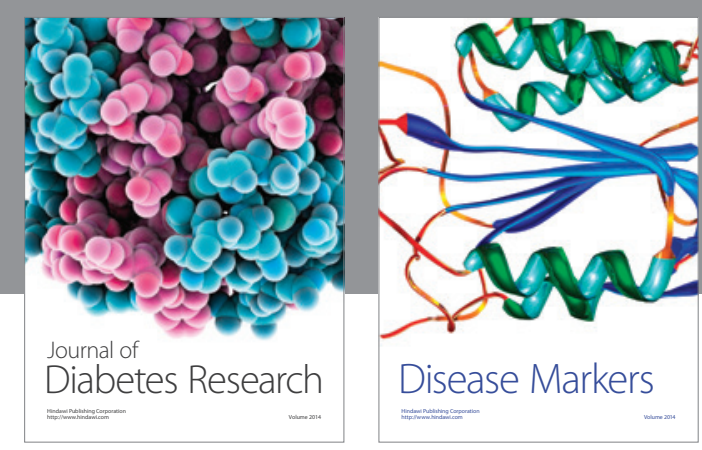

Disease Markers
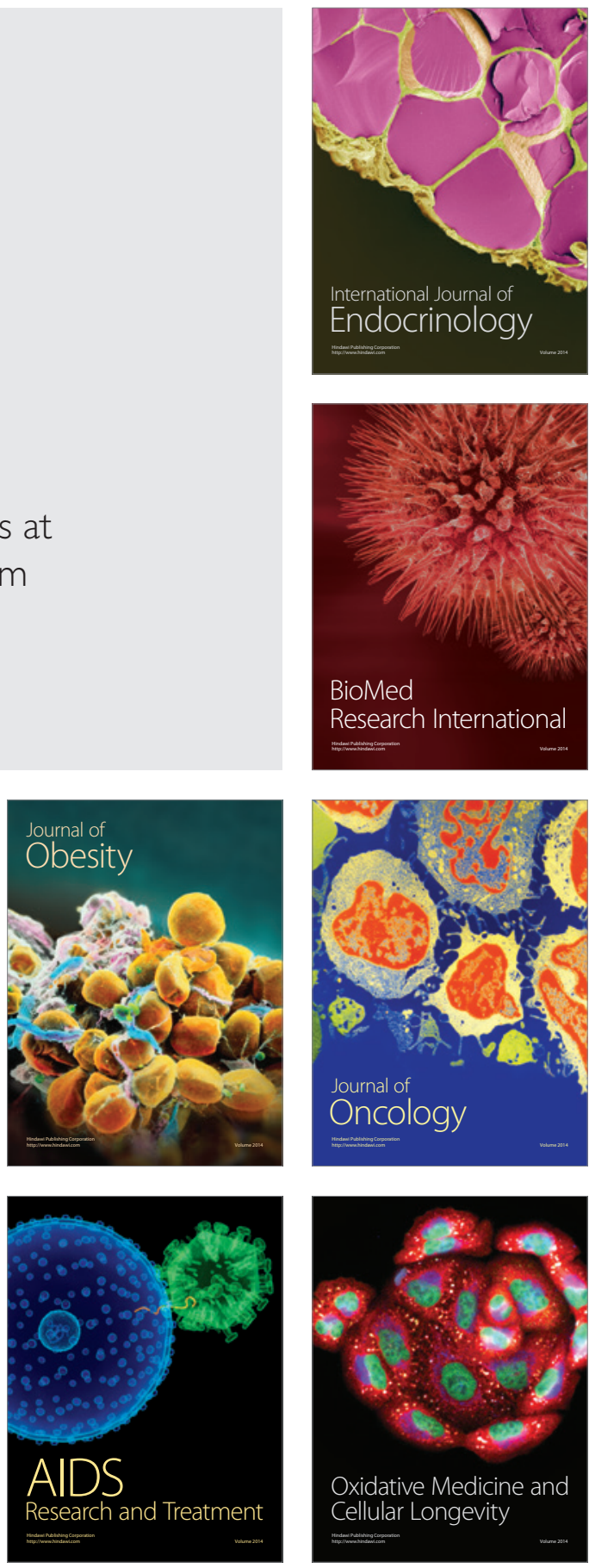